\title{
La investigación como fundamento para el diseño de la Licenciatura en inglés como lengua extranjera de la UNAD'
}

Nélida Cárdenas Vergaño ${ }^{2}$

\begin{abstract}
Resumen
Este artículo presenta el resultado de un proceso investigativo, que permitió el diseño curricular de un programa en la modalidad a distancia-virtual, desde el proyecto UNAD-PG-03-2009: Diseño curricular de la Licenciatura en Inglés como Lengua Extranjera 'LILE', del grupo de investigación Gestión Vital y la Línea Visibilidad y Gestión del Conocimiento, de la Escuela Ciencias de la Educación (ECEDU) de la Universidad Nacional Abierta y a Distancia (UNAD).
\end{abstract}

Palabras clave: formación y/o educación a distancia, inglés, metodología, plan de estudios. 


\title{
Research as a basis for designing a degree program in english as foreign language of UNAD
}

\begin{abstract}
This paper shows the result of a research process from which the curricular design of a program in distance learning and virtual methodology was designed; this research was developed from the project UNAD-PG-03-2009: "Curriculum design of a Degree Program in English as Foreign Language”, from Gestión Vital group and Visibilidad y Gestión del Conocimiento Research Line, from the School of Educational Sciences (ECEDU) at the Universidad Nacional Abierta y a Distancia (UNAD-Colombia).
\end{abstract}

Keywords: Curriculum, Distance Education, English, Methodology.

Recibido: 14-04-2014

Aceptado: 07-05-2014

\section{Contextualización}

El dominio del inglés responde a la demanda nacional colombiana e internacional de formar ciudadanos del mundo que puedan comunicarse a través de diversos medios, directa o indirectamente, es decir, utilizando las herramientas tecnológicas desde la virtualidad; al mismo tiempo, permite que los estudiantes, docentes y el resto de la comunidad académica tengan acceso a los avances de la ciencia y la tecnología.

Igualmente, el fortalecer la enseñanza y el aprendizaje de una lengua extranjera en Colombia ha sido una preocupación manifestada desde el Ministerio de Educación Nacional, con el fin de generar dinámicas nacionales de tipo académico, cultural y económico, entre otros. «El manejo de una segunda lengua significa poderse comunicar mejor, abrir fronteras, comprender otros contextos, apropiarse de saberes y hacerlos circular, entender y hacernos entender, enriquecerse y jugar un papel decisivo en el desarrollo del país. Ser bilingües es tener más conocimientos y oportunidades para ser más competentes y competitivos y mejorar la calidad de vida de todos los ciudadanos» (MEN, 2005). 
Es por esto que la UNAD, desde su perspectiva de consolidación del proceso de internacionalización, posibilita visionar a un maestro colombiano capaz de proponer creativamente a la problemática que surge del ejercicio profesional en la docencia del inglés en un contexto nacional e internacional diverso. La formación de profesionales críticos, creativos y propositivos con un amplio dominio del inglés se constituye en el foco de preparación que permite contribuir a la reconstrucción social del país, donde el aprecio por la identidad, la interculturalidad, la multiculturalidad y valores como la tolerancia y la solidaridad, así como el conocimiento de otras culturas, son primordiales.

Se hizo necesario entonces responder a las propuestas y políticas nacionales respecto al inglés, con el propósito de formar docentes que influenciaran directamente a estudiantes de educación preescolar, básica, media y superior, capaces de responder a un nuevo entorno bilingüe, pero sin perder de vista la promoción y protección de otras lenguas en poblaciones étnicas, raizales y de frontera. Por lo tanto, se gestó el proceso investigativo desde la Escuela Ciencias de la Educación de la UNAD, para el diseño curricular de un programa donde el área específica fuera el inglés y que a la vez tuviera un alto contenido en el manejo de las Tecnologías de la Información y la Comunicación.

La pregunta de investigación planteada desde el proyecto UNAD-PG-03-2009 fue: ¿Cuáles son las principales características y parámetros que se deben tener en cuenta para el diseño curricular de una Licenciatura en Inglés como Lengua Extranjera en la modalidad a distancia-virtual?, en tanto que los objetivos planteados llevaron a la construcción del diseño curricular de la Licenciatura en Inglés como Lengua Extranjera en la modalidad de Educación a Distancia virtual, mediante el análisis del estado del arte del proceso de enseñanza-aprendizaje de esa lengua, la visualización de la cobertura de la oferta a nivel nacional e internacional y su diseño con base en la normatividad nacional colombiana.

\section{Metodología}

Desde la pregunta de investigación y la normatividad del Ministerio de Educación $\mathrm{Nacional}^{3}$, se procedió a organizar las diferentes etapas para el desarrollo del documento maestro que sería presentado y sustentado ante el MEN y que tendría la descripción de cada una de las características asociadas al registro calificado del programa de Licenciatura en Inglés como lengua extranjera. A continuación se presentan las generalidades abordadas para el desarrollo curricular desde el proceso investigativo con un enfoque interpretativo-descriptivo. 


\section{Estado de la educación en inglés como lengua extranjera en el ámbito colombiano ${ }^{4}$}

En esta etapa el equipo investigador realizó una revisión general de las características de los programas profesionales en el área de inglés ofertados en el país, para proceder luego a su análisis y permitir así una visión detallada del estado de la enseñanza del área específica, encontrando lo siguiente:

\section{Oferta colombiana de programas de licenciatura en idiomas ${ }^{5}$}

Desde el panorama nacional, se encontró la presencia de 1.229 programas de licenciaturas en educación, cuyas denominaciones correspondían a diferentes áreas del conocimiento en corresponsabilidad con las áreas obligatorias y fundamentales previstas en la educación básica y media. El 92\% de tales programas contaban con profundización en áreas distintas al campo de formación del inglés como lengua extranjera. Esto indica que solo el $8 \%$ de los programas (98 en total) correspondía particularmente a los campos de formación en castellano e idiomas extranjeros, estos últimos específicamente inglés y francés.

\section{Oferta educativa en Colombia}

Licenciaturas área distintas a los idiomas extranjeros

Licenciaturas para la formación en idiomas

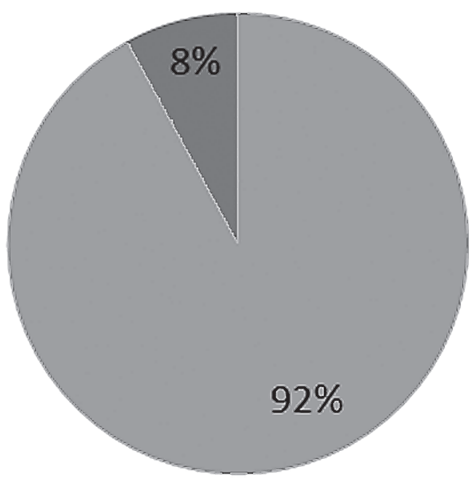

Figura 1. Oferta educativa en Colombia Fuente: LILE-UNAD, 2010.

\footnotetext{
4. Para el desarrollo de este apartado participó el equipo de diseño curricular de la Licenciatura, conformado por los docentes Farides Pitre Redondo y Alexander Fernández.

5. Información recopilada en febrero de 2010 a través del Sistema Nacional Instituciones Educación Superior - SNIES del Ministerio de Educación Nacional (Colombia).
} 
De la misma forma, en el área específica de humanidades e idiomas se encontró una oferta de programas de licenciatura: el 48\% corresponde al énfasis en humanidades, lenguas castellana e inglés, el 19\% a licenciaturas en educación básica con énfasis en lengua castellana, y el 33\% con énfasis en humanidades, lengua castellana e idiomas extranjeros.

También se encontró que solamente estaba activo el 50\% de los programas, o sea que únicamente 49 licenciaturas con énfasis en humanidades, lengua castellana, idiomas extranjeros o áreas afines estaban siendo ofertados en el país, el otro $50 \%$ estaba inactivo. De estos 49 programas el $84 \%$ correspondía al nivel de formación del inglés con otras lenguas y solo el 16\% ofrecía un nivel de profundización en la lengua inglesa, por lo tanto el panorama del estado de formación era deficiente, que no se ajusta a las necesidades de formación que requiere Colombia como país competitivo con proyección de apertura a mercados globales.

En cuanto a la metodología educativa de oferta, se encontró que el 90\% de los 49 programas que contaban con oferta vigente estaban orientados bajo la metodología de educación presencial, el $8 \%$ en la modalidad distancia tradicional, mientras que el $2 \%$ (1 programa) era ofrecido virtualmente. Igualmente, el escenario de oferta de los programas estaba conformado así: el 55\% en universidades privadas y el $45 \%$ en universidades estatales.

\section{Criterios generales de formación de las licenciaturas colombianas ${ }^{6}$}

Desde la misma revisión realizada en 2009 y 2010, se tuvieron en cuenta varios criterios comunes de los programas ofertados en el país, que llevaron a una consolidación de los parámetros a tener en cuenta durante el diseño de la Licenciatura de la UNAD; algunos de esos criterios fueron los objetivos de formación, la formación por ciclos o campos de formación y el énfasis puesto en el idioma inglés. A continuación se presentan las generalidades del análisis de este aspecto.

Objetivos de formación de las licenciaturas. En el escenario colombiano los programas de licenciatura en inglés buscan la formación, actualización y perfeccionamiento de estudiantes desde el desarrollo de conocimientos, habilidades y destrezas con la calidad humana y ética como resultado del proceso de formación integral.

Perfiles y competencias a desarrollar. En este aspecto se evidenció una alta descripción de las competencias disciplinares e interdisciplinares que constituyen el saber del inglés como lengua extranjera. Las dimensiones de la formación 
integral de acuerdo con el rol de docente como teórico de la lengua, agente de un proceso cultural, y se destaca la consideración de ver al futuro docente como guía pedagógico de sus estudiantes.

Formación por ciclos. Un bajo porcentaje de los programas (18\%) contaba con su plan de estudios dividido por ciclos de formación, algunos de los ciclos encontrados son: ciclo de fundamentación o fundamentación conceptual, de contextualización, de profundización, disciplinar, de innovación y creación, de libre elección, de énfasis y/u optativo. En otras universidades encontramos núcleos de formación tales como lengua y educación, pedagogía y enseñanza del inglés, desarrollo de la lengua extranjera en el aula, el papel de la lengua extranjera inglés y la formación cultural. Las otras universidades $(82 \%)$ presentaban su plan de estudios dividido en semestres.

Campos de formación. El $90 \%$ de la universidades presenta su plan de estudios dividido en campos de formación, entre ellos están: campo pedagógico, pedagógico/didáctico, científico, disciplinar, humanístico, investigativo, lengua materna, formación en lingüística, interdisciplinar/seminarios interdisciplinarios y/o de profundización, formación básica, formación integral, comunicativo/ estético, ético/político, ético y humanístico; solo una universidad (el 2,7\%) posee como campo de formación el apoyo en tecnologías. Lo anterior no implicaba que no se contemplaran cursos específicos con componente tecnológico durante las carreras.

Inglés. Se encontró que el $90 \%$ de las universidades contaba con 6 niveles de lengua, además de profundización en inglés en los últimos semestres de las diferentes licenciaturas; dicha profundización era en habilidades comunicativas, literatura, traducción de textos, diseño de textos, composición escrita y conversacional del inglés, además de saberes específicos tales como: morfosintaxis, fonética, fonología, morfología, cultura y sociedad.

Créditos académicos. En este componente se argumentan el tiempo de estudio y el tiempo de acompañamiento al estudiante, los cuales en su totalidad oscilan entre 150 y 180 créditos por programa y entre 8 y 10 semestres.

Denominaciones. La versatilidad en las denominaciones existentes de estos programas de licenciatura, apunta a combinar la enseñanza del inglés, la enseñanza de la lengua castellana y la enseñanza de otro idioma (ya sea francés o lengua clásica), esto disminuye considerablemente el nivel de aprehensión y comprensión de al menos una de las lenguas allí determinadas. El énfasis puesto en el idioma inglés no necesariamente es prioritario puesto que el estudiante debe aprenderlo simultáneamente con otras ofertas, lo que imposibilita el dominio consciente de una lengua en particular. 
Después de realizar la revisión del estado de los programas afines al inglés en Colombia y en algunos programas extranjeros (Argentina, México, Chile, Venezuela), se pasó a la definición de los aspectos teóricos, los atributos o factores distintivos que caracterizaran el programa de la UNAD, las competencias a desarrollar por parte del estudiante y futuro licenciado, la distribución curricular por campos de formación y la innovación en la distribución de los cursos por microcurrículos, con el fin de hacer énfasis en las áreas de conocimiento específicas del programa.

\section{Fundamentación teórica para el desarrollo de la propuesta curricular}

La Licenciatura en Inglés como lengua extranjera centró su fundamentación teórica en la propuesta del Proyecto Académico Pedagógico Solidario (PAPS) 2008 y su respectiva actualización en 2010. Además, se tuvieron en cuenta aspectos relevantes desde el área Ciencias de la Educación como referentes en la construcción del programa ${ }^{7}$.

Según Elkind (2004), el constructivismo en todas sus diferentes formas es una filosofía y pedagogía educativa importante. Lo que las diversas interpretaciones del constructivismo tienen en común es la propuesta de que el estudiante es un participante activo en la construcción de la realidad y no sólo un grabador pasivo de la misma. El constructivismo hace eco de la filosofía implícita en el Emilio de Rousseau (2005), donde se sostiene que los estudiantes tienen sus propias formas de conocimiento y que estas deben ser valoradas y respetadas. También refleja la filosofía kantiana (Kant, 2002), respecto a la resolución de la controversia naturaleza / crianza. Kant sostiene que la mente establece las categorías de conocimiento mientras que la experiencia proporciona el contenido. Entre tanto, Piaget (1950), creó la versión contemporánea del constructivismo, demostrando que las categorías de conocimiento, no menos que los contenidos del conocimiento, se construyen en el curso del desarrollo.

Así mismo, se toma la pedagogía activa por cuanto se concibe la educación como un señalamiento de caminos para la auto-determinación personal y social y para el desarrollo de la conciencia crítica, por medio de la cual el análisis y la transformación de la realidad acentúa el carácter activo del estudiante en el proceso de aprendizaje, apropiando los conocimientos mediante una articulación de la teoría con la práctica.

Entonces, la pedagogía activa identifica al docente como un guía, orientador, catalizador y animador de este proceso y asocia el aprendizaje con el desarrollo de búsqueda de significados, críticas, invención e indagación en contacto 
permanente con la realidad. Así, se concibe la verdad como un proyecto elaborado y no como un elemento en posesión de pocas personas, y la relación docenteestudiante como un proceso de diálogo, cooperación y apertura permanente.

La pedagogía cognitiva toma su aporte fundamental en la concepción de que el aprendizaje debe ser una actividad significativa para la persona que aprende y tener no solo relación entre el conocimiento nuevo y el que ya posee el estudiante, sino también interpretando el concepto de aprendizaje como una actividad social, ya que en ocasiones el estudiante aprende de forma más eficaz cuando lo hace en un contexto de colaboración e intercambio con sus compañeros (Díaz y Hernández, 1999). Por lo anterior, las prácticas y el desarrollo de trabajos en equipo son estrategias de formación del Programa de Licenciatura en Inglés como lengua extranjera de la UNAD.

En el modelo andragógico, la experiencia es siempre el punto de partida del proceso educativo, nunca el resultado, y por ello se fundamenta en las siguientes premisas (Knowles, 1970):

- La necesidad de saber. Los estudiantes están dispuestos a aprender lo que necesitan saber y son capaces de hacer, con el propósito de enfrentar las situaciones de la vida real.

- El autoconcepto de los estudiantes. Es de resaltar que se debe promover en los estudiantes el autoconcepto de seres responsables de sus propias acciones y de su propia vida.

- El papel de las experiencias. Los estudiantes llegan a una actividad educativa con un volumen y una calidad de experiencias, tendiendo a formar hábitos mentales y prejuicios que cierran la mente a las ideas nuevas, percepciones frescas y otras formas de pensar.

- Características básicas del ambiente educativo. Algunos elementos esenciales son el respeto por la personalidad, participación en la toma de decisiones, libertad de expresión, responsabilidad mutua para definir metas, planeación, conducción y evaluación de actividades.

Los estudiantes en la modalidad de educación a distancia deben tener la condición de aprender autónomamente en el sentido de construir sus conocimientos desde la independencia y autonomía en aras de conseguir el éxito académico, al punto que se ven en la necesidad de aprender ya no en un momento de la vida sino de manera permanente, estructurando formas de aprendizaje en las que puedan fijar sus propias normas y así darles cumplimiento.

- Educación a distancia. Para la UNAD, la educación a distancia está prevista dentro del contexto formativo de la educación permanente, porque reconoce 
que los procesos formativos de la persona y los procesos productivos del conocimiento duran toda la vida, lo mismo que la capacidad para aprender y para la autoconstrucción individual y colectiva, a partir de la autonomía mental, ética, intelectual y moral de los sujetos protagónicos de su aprendizaje, la cual reconoce que existen diferentes estilos cognitivos, condiciones de aprendizaje, ritmos para aprender, privilegiando las estructuras de participación, no sólo de los estudiantes sino de las comunidades regionales y locales para que intervengan en la identificación y solución de sus necesidades y se comprometan en el diseño y realización de sus propios proyectos educativos, culturales, tecnológicos y socio-productivos.

La educación abierta y a distancia se desarrolla fundamentalmente como un sistema social abierto, de carácter holístico, en continua interacción con el entorno socio-cultural y regido por los principios sistémicos de adaptación, retroalimentación, organización y relación de elementos, dinámica de procesos y coherencia entre medios y fines. Reconoce a su vez que el centro del aprendizaje es el estudiante, quien debe responder por la gestión de su formación a partir de la apropiación crítica de la realidad, del desarrollo de su propio potencial de aprendizaje y de la capacidad de autodeterminación, autocontrol y autogestión, que fundamenta el aprendizaje autónomo y la autogestión formativa.

La educación abierta y a distancia asume diseños curriculares pertinentes y flexibles, en la medida en que los campos disciplinarios de los programas responden a las condiciones de los contextos y de los estudiantes, mediante una estructura ágil, para posibilitar el avance a su propio ritmo, en un tiempo académico razonable y socialmente pertinente, independiente del espacio físico de aprendizaje y de la edad escolar, utilizando múltiples mediaciones pedagógicas, derivadas de los medios de comunicación y de las tecnologías telemáticas; lo mismo que mediadores referidos a los docentes, tutores y consejeros como su cuerpo académico y asesores, grupos de estudiantes, equipos de trabajo, asociaciones profesionales y gremiales, entre otros que tienen la función de facilitar el ingreso y garantizar la permanencia de los estudiantes, sin importar el lugar en donde se encuentren.

Esta condición de aprender autónomamente significa saber superar los obstáculos o limitaciones que se puedan presentar cuando se espera alcanzar, lograr, comprender y aprehender un objeto de conocimiento determinado. Para ello el estudiante requiere utilizar capacidades y competencias básicas, como la memoria y la atención, la lectura, saber escuchar, saber re-escribir, comprender, repasar, programar el aprendizaje, la concentración y la autosuficiencia, y con ello tomar la iniciativa de solucionar sus necesidades y la búsqueda de recursos que le permitan construir sus aprendizajes.

Castro (2006), expresa que el aprendizaje autónomo es permanente, que requiere de autodirección y autocontrol, por tanto, exige de actividades conscientes, 
planificadas e intencionales para el logro de objetivos propuestos, además de espacios de reflexión que permitan procesos de autoevaluación. Este tipo de aprendizaje convierte al estudiante en un autorrealizador de su formación, capaz de definir sus metas, de seleccionar los medios y procedimientos que le permitirán alcanzar los logros, establecer tiempos y condiciones de aprendizaje, además de controlar los resultados a partir de su propia determinación.

Aebli (2001), hace referencia a que el aprendizaje autónomo se extiende también a los problemas de la psicología social y de la psicología de la personalidad. No todo aprendizaje autónomo es de por sí aprendizaje aislado. Por el contrario, si los sujetos continúan aprendiendo por fuera de la escuela, ello se debe a que casi siempre se integran a grupos y asociaciones que les permite colaborar y aprender en el trabajo en común. Quien se encuentra en situación de aprendizaje, debe lograr inicialmente unos rendimientos que implican competencia social y la motivación correspondiente; establecer contactos con los miembros de tales grupos, indagar cómo les va, no solo objetiva sino también interpersonalmente. Se considera, por tanto, que también se prepara a una persona al aprendizaje autónomo en la medida en que se le capacita y motiva a tomar parte y trabajar en un grupo.

El aprendizaje autónomo es un proceso de apropiación crítica de la experiencia vital, intelectual y cultural, a partir del reconocimiento de la realidad personal y social, mediante la profundización teórica de conceptos básicos, principios explicativos y valores fundamentales, generados en forma metódica, sistemática y autorregulada, para transferirlos comprensivamente a diferentes contextos y aplicarlos creativamente en la solución de problemas de la vida cotidiana, en el desarrollo de procesos académicos y en la promoción del desarrollo humano (UNAD, 2011).

Tomando el constructivismo, la pedagogía activa, cognitiva, la andragogía y el aprendizaje autónomo como fundamentos pedagógicos del programa, cada módulo de los cursos define los propósitos de formación y su articulación con los perfiles ocupacional y profesional de los participantes.

Dentro de la fundamentación metodológica es de mencionar también la utilización de las Tecnologías de Información y Comunicación (TIC) desde los medios y mediaciones tecnopedagógicas como herramientas para la creación de aprendizajes.

\section{Atributos o factores que constituyen los rasgos distintivos del programa}

La Licenciatura en Inglés como lengua extranjera de la Universidad Nacional Abierta y a Distancia (en coherencia con el modelo pedagógico), involucra clara 
y particularmente el desarrollo de competencias comunicativas en el idioma, el desarrollo de conocimiento, habilidades y actitudes desde: 1) El autoaprendizaje como componente esencial para el desarrollo de cada una de las actividades programadas en los diferentes cursos académicos; 2) Su alto contenido en el uso de las Tecnologías de la Información y la Comunicación; 3) La investigación como eje transversal en el proceso de aprendizaje; y 4) La metodología a distancia y virtual, que permite el acceso a poblaciones lejanas que no tienen posibilidad de movilizarse a las diferentes instituciones.

\section{Competencias a desarrollar por parte de los estudiantes}

Esa nueva propuesta curricular consideró importante dentro de su diseño puntualizar en la descripción de las competencias que estarían inmersas en el proceso de enseñanza y aprendizaje del inglés como lengua extranjera, previstas como «el conjunto de saberes, conocimientos, destrezas y características individuales que permiten a los estudiantes (personas) realizar acciones en un contexto determinado» (MEN, 2006).

Desde esa óptica, para la propuesta se concibió, entonces, un enfoque comunicativo de la lengua inglesa centrado en el estudiante, donde se propiciara su participación en procesos de autorregulación y de autoaprendizaje. Este enfoque permitiría al estudiante dar cuenta de la competencia comunicativa, la cual implica «un saber/hacer flexible, que se actualiza en contextos significativos y que supone la capacidad para usar los conocimientos acerca de la lengua en diversas situaciones, tanto dentro como fuera de la vida escolar» (MEN, 2006). Igualmente, permitiría a los estudiantes desarrollar habilidades y saberes que se relacionan con las dimensiones ética, estética, social y cultural de la lengua que se aprende. Esto se traduce a las posibilidades reales para comprender e interpretar la realidad. De esa manera, se tuvo en cuenta como pilar educativo del programa: «aprender a conocer, aprender a hacer, aprender a vivir juntos y aprender a ser» (MEN, 2006). Desde la definición por competencias se contemplaron tres aspectos que a su vez forman parte del aprendizaje para toda la vida: a) Actuar de forma autónoma, b) Usar herramientas interactivas, y c) Funcionar en grupos sociales heterogéneos.

\section{Campos de formación}

Los campos y áreas de formación que se asumieron para el diseño del Programa de Licenciatura en Inglés como lengua extranjera, fueron tomados con base en la propuesta del PAPS-UNAD 2008, así:

*Formación Básica Común. El principio de racionalidad pedagógica se evidencia en la política de la introducción de los estudios generales a partir 
de la formación básica común de dos de las áreas o campos de formación que estructuran la organización curricular de los diferentes programas: la formación socio-humanística y la formación investigativa.

*Formación Sociohumanística. Tiene como propósito contribuir a la formación del estudiante en calidad de persona, en sus dimensiones psicológica, ética y comunicativa, y a la formación de procesos de aprendizaje autónomo. La formación socio humanística es obligatoria para todos los programas que ofrece la UNAD y se constituye en uno de los aspectos esenciales del sello formativo que imparte; por lo tanto, cursos como Competencias comunicativas, Cultura política, Ética, Sociología, Antropología y Psicología, fueron tenidos en cuenta dentro de este campo de formación.

*Formación investigativa. Tiene como propósito fomentar el desarrollo del espíritu científico entre los estudiantes en los diversos programas de la institución, a la vez que se apropian de herramientas básicas en torno a los procesos investigativos, con el fin de agregar valores tanto en la formación personal como en la búsqueda sistemática de alternativas diversas para la resolución de problemas específicos. La formación investigativa adquiere un carácter básico y obligatorio para los estudiantes matriculados en los diferentes programas de pregrado y postgrado. En la Licenciatura en inglés se planteó trabajar, desde el componente básico común, cursos como: Estadística Descriptiva, Metodología y Técnicas de la Investigación y Epistemología, que le permitirán al estudiante contextualizarse en el área investigativa y que al realizar cursos como Teacher Development, Educational Research y Research Project profundice en este aspecto y al mismo tiempo pueda realizar un proceso investigativo al finalizar su licenciatura.

*Formación Básica Disciplinar. Son las áreas (comunes y específicas) propias de la disciplina del inglés como lengua extranjera y de pedagogía, que le permitirán al profesional conocer, interiorizar y aplicar principios, teorías y definiciones para el uso del inglés en contextos específicos de aprendizaje, además de conocer e identificar las particularidades culturales de la lengua y de los principios generales de la pedagogía. Los cursos que el estudiante recibirá en este componente son: Pedagogía, Teorías del aprendizaje, Teorías Curriculares, Diseño de instrucción, Evaluación y Didáctica. Igualmente, se tendrán en cuenta tres cursos de fortalecimiento en Lengua Materna y Raíces Griegas y Latinas, como base para generar un mejor aprendizaje de la lengua extranjera inglés. Por otro lado, y con el fin de contextualizar a los estudiantes en el aprendizaje, los métodos de enseñanza de una lengua extranjera y el uso de la tecnología para la enseñanza y el autoaprendizaje de dicha lengua, se tendrán en cuenta cursos tales como: Teaching English to Adolescents and Adults, Methods of Teaching English as a Foreign Language and Technology in Teaching English as a Foreign Language. 
*Formación Profesional Especifica. Son aquellas áreas que permitirán adquirir destrezas, habilidades y competencias para el quehacer propio de la disciplina y tener la capacidad de llevarlas a cualquier contexto educativo en la metodología específica. Esto le permitirá al licenciado en inglés de la UNAD desempeñarse como docente, tutor, asesor, investigador e integrante de grupos interdisciplinarios en los distintos campos del saber. En este aspecto se encuentran incorporados los ocho niveles de inglés para la adquisición y el aprendizaje de lengua, finalizando con énfasis en English conversation y Academic writing. De la misma manera, cursos como Introducción a la Licenciatura en Inglés como lengua extranjera, Phonetics and phonology, First and second language acquisition and learning, Language forms and functions permitirán a los estudiantes reconocer las características particulares del programa de licenciatura $\mathrm{y}$, paralelamente, mejorar sus competencias comunicativas para su futura experiencia pedagógica. Igualmente, los estudiantes tendrán fortalezas en el diseño de materiales con base en los diferentes ambientes de enseñanza: Materials design for virtual environments y Materials design for face to face environments. Los cursos tenidos en cuenta para estructurar competencias pedagógicas y didácticas son: Teacher development, Testing and evaluation in ELT, Didactics of english, Pedagogical experience I, Pedagogical experience II, Educational research y Research project.

*Electivas. Del 100\% de los créditos electivos (40), los estudiantes tendrán a su disposición un $65 \%$ de créditos electivos propios del programa para el fortalecimiento de sus competencias en la licenciatura; a la vez contarán con un $35 \%$ de créditos electivos en otros programas de pregrado de la UNAD.

\section{Estrategias para el seguimiento de las actividades comunicativas de lengua}

Para el desarrollo de las actividades de recepción y de producción en inglés, existirá un seguimiento detallado al proceso del estudiante por parte del tutor; por lo tanto, desde la Escuela Ciencias de la Educación, en el momento de contratar a los tutores se plantearán las tareas a desarrollar, donde se evidencien los momentos de sincronía (acompañamiento) y las actividades asincrónicas, con el fin de que los estudiantes desarrollen sus competencias comunicativas en inglés.

A nivel general, para desarrollar las actividades de lengua a llevar a cabo en los cursos de inglés de la Licenciatura, los estudiantes recibirán capacitación previa sobre el uso de las herramientas de comunicación sincrónicas, tales como Skype, Webconference, entre otros, y se les exigirá participar en cada una de las sesiones programadas en un cronograma establecido al inicio de cada curso. Igualmente, las actividades se diseñarán con base en el nivel de lengua que 


\section{Nélida Cárdenas Vergaño}

La investigación como fundamento para el diseño de la Licenciatura en inglés como lengua extranjera de la UNAD. Artículo producto de la investigación.

se encuentren cursando los estudiantes. En la siguiente tabla se describen las actividades propuestas para el acompañamiento sincrónico y asincrónico en el desarrollo de las cuatro habilidades comunicativas.

\begin{tabular}{|c|c|c|c|c|}
\hline $\begin{array}{c}\text { Tipo de } \\
\text { Actividad / } \\
\text { Habilidad }\end{array}$ & $\begin{array}{l}\text { Escuchar - Comprensión } \\
\text { Auditiva (input) }\end{array}$ & $\begin{array}{l}\text { Leer - Comprensión } \\
\text { Lectora (input) }\end{array}$ & $\begin{array}{c}\text { Hablar - Expresión Oral } \\
\text { (output) }\end{array}$ & $\begin{array}{l}\text { Escribir - Expresión } \\
\text { Escrita (output) }\end{array}$ \\
\hline Sincrónica & $\begin{array}{c}\text { Vía Skype o } \\
\text { Webconference: Escuchar: } \\
\text { las instrucciones dadas } \\
\text { por el tutor, la información } \\
\text { brindada por sus } \\
\text { compañeros, conferencias, } \\
\text { presentaciones. }\end{array}$ & $\begin{array}{l}\text { Vía Skype o } \\
\text { Webconference: Leer en } \\
\text { voz alta, leer para seguir } \\
\text { instrucciones, lectura } \\
\text { general en grupos } \\
\text { colaborativos. }\end{array}$ & $\begin{array}{l}\text { Vía skype o } \\
\text { webconference: Describir } \\
\text { elementos visuales, leer } \\
\text { en voz alta, representar } \\
\text { roles asignados } \\
\text { previamente, hablar sobre } \\
\text { temas libres, cantar, } \\
\text { participar en debates, } \\
\text { hablar a los compañeros. }\end{array}$ & $\begin{array}{c}\text { Vía skype o } \\
\text { webconference: } \\
\text { Tomar notas, tomar } \\
\text { mensajes de dictados, } \\
\text { completar formularios } \\
\text { o cuestionarios. }\end{array}$ \\
\hline Asincrónica & $\begin{array}{c}\text { Desde el curso de la } \\
\text { plataforma o como } \\
\text { material adicional: } \\
\text { Escuchar retransmisiones } \\
\text { y material grabado, } \\
\text { instrucciones, conferencias, } \\
\text { presentaciones, } \\
\text { conversaciones entre } \\
\text { hablantes nativos, } \\
\text { información de medios } \\
\text { de comunicación (radio, } \\
\text { televisión, grabaciones, } \\
\text { cine), todo el material } \\
\text { brindado en el curso. }\end{array}$ & $\begin{array}{l}\text { Desde el curso de la } \\
\text { plataforma o como } \\
\text { material adicional: Leer } \\
\text { el material propuesto } \\
\text { en el curso, para seguir } \\
\text { instrucciones, cuentos, } \\
\text { historias narrativas, } \\
\text { textos de consulta, } \\
\text { material producido } \\
\text { por el tutor y por sus } \\
\text { compañeros, mensajes, } \\
\text { cartas, comunicados. }\end{array}$ & $\begin{array}{l}\text { Desde el curso de la } \\
\text { plataforma o como } \\
\text { material adicional. } \\
\text { Grabaciones sobre: } \\
\text { lecturas a textos previos, } \\
\text { describir con base en } \\
\text { esquemas, imágenes o } \\
\text { gráficos, preparación de } \\
\text { monólogos, descripción } \\
\text { de experiencias, } \\
\text { entrevistas. }\end{array}$ & $\begin{array}{l}\text { Desde la plataforma } \\
\text { o como material } \\
\text { adicional: Completar } \\
\text { formularios o } \\
\text { cuestionarios, producir } \\
\text { presentaciones para } \\
\text { exponer oralmente, } \\
\text { tomar notas, escribir } \\
\text { textos narrativos, } \\
\text { cartas (formales } \\
\text { e informales), } \\
\text { informes académicos, } \\
\text { mensajes. }\end{array}$ \\
\hline $\begin{array}{l}\text { Aspectos a tener } \\
\text { en cuenta }\end{array}$ & $\begin{array}{l}\text { Al estudiante se le explicará } \\
\text { previamente el fin de cada } \\
\text { una de las actividades, } \\
\text { recibirá información con } \\
\text { base en su nivel y se } \\
\text { espera que él participe en } \\
\text { cada una de las tareas } \\
\text { propuestas, tanto desde la } \\
\text { plataforma como desde las } \\
\text { actividades extras. }\end{array}$ & $\begin{array}{l}\text { El estudiante recibirá } \\
\text { técnicas de lectura } \\
\text { y comprensión de } \\
\text { la misma, deberá } \\
\text { asistir a las sesiones } \\
\text { grupales programadas } \\
\text { para el desarrollo de } \\
\text { las tareas y tendrá } \\
\text { acompañamiento } \\
\text { permanente por parte } \\
\text { del tutor. }\end{array}$ & $\begin{array}{c}\text { El estudiante se } \\
\text { capacitará en el uso de } \\
\text { las herramientas (Skype, } \\
\text { webconference) para } \\
\text { el desarrollo de estas } \\
\text { actividades y se le exigirá } \\
\text { estar en cada una de las } \\
\text { sesiones programadas } \\
\text { por el tutor. }\end{array}$ & $\begin{array}{c}\text { El estudiante } \\
\text { conocerá previamente } \\
\text { la finalidad de } \\
\text { sus escritos, se le } \\
\text { brindarán ejemplos } \\
\text { y se le asesorará } \\
\text { permanentemente } \\
\text { por el parte del tutor, } \\
\text { se le exigirá cumplir } \\
\text { con todas actividades } \\
\text { programadas. }\end{array}$ \\
\hline
\end{tabular}

Tabla 1. Actividades sincrónicas y asincrónicas de la Licenciatura en Inglés.

Fuente: LILE, UNAD, 2010.

\section{Espacios académicos de la experiencia pedagógica}

Siendo un programa de Licenciatura en Inglés como lengua extranjera, los estudiantes deberán desarrollar su experiencia pedagógica después de haberse preparado a través de los diferentes cursos del programa, específicamente de los preparatorios para la misma, así: Teacher Development, Pedagogical Experience I y II. 


\section{Promoción de la formación investigativa}

Dentro del plan de estudios de la licenciatura, la investigación jugará un papel esencial y creativo para la labor educativa, que permitirá optimizar los procesos de enseñanza-aprendizaje desde el acercamiento al aprendizaje autónomo, a las realidades sociales y la generación de dinámicas que favorezcan el desarrollo humano y social, la construcción de ciudadanía y la existencia de escenarios posibles para el verdadero ejercicio de la democracia.

En la Licenciatura en Inglés se proyectan cursos de contextualización en investigación, tales como Metodología y técnicas de la investigación, Epistemología y Estadística Descriptiva, para luego abordar la profundización en el área con cursos como: Educational Research, Research Project, complementados por el curso de Teacher Development que lleva a los estudiantes a hacer una visualización detallada de los procesos pedagógicos que puede investigar a futuro.

Desde su propio diseño, la Licenciatura en Inglés como lengua extranjera se articuló a la línea de investigación Bilingüismo en la educación a distancia mediada por tecnologías, de la Escuela Ciencias de la Educación de la UNAD; esta línea busca diseñar e Implementar propuestas, tanto académicas como de extensión, con el fin de llevar un seguimiento detallado de los aportes institucionales en esta área al Plan Nacional de Bilingüismo, teniendo como base el Proyecto Académico Pedagógico Solidario de la UNAD. Igualmente, se estableció que los semilleros de investigación se vincularán a Virtualex, grupo de investigación de la Licenciatura en Inglés, que tiene como objeto generar nuevo conocimiento sobre la relación bilingüismo y procesos de enseñanza-aprendizaje con énfasis en e-learning, para fortalecer el desarrollo de competencias tanto lingüísticas como de acción pedagógica con perspectivas locales y globales desde los fundamentos del Proyecto Académico Solidario de la UNAD, los lineamientos de orden nacional y el marco de referencia europeo para el aprendizaje de lenguas.

\section{Situación actual del programa}

La Licenciatura en Inglés como Lengua Extranjera de la UNAD, recibió su Registro Calificado por siete (7) años mediante la Resolución 3286 del 25 de abril de 2011.

\begin{tabular}{c|c|}
\hline $\begin{array}{c}\text { Nombre del programa: } \\
\text { Código SNIES: }\end{array}$ & Licenciatura en Inglés como Lengua Extranjera \\
\hline Registro Calificado: & 91276 \\
\hline Estudiantes matriculados a 2013 I & Resolución 3286 del 25 de abril de 2011 \\
\hline & $\begin{array}{c}\text { Tabla 2. Registro calificado LILE. } \\
\text { Fuente: Producción propia. }\end{array}$ \\
\hline
\end{tabular}


El programa inició en 2011-2 con 310 estudiantes matriculados; a 2013-2 cuenta con 1281 matriculados. La Licenciatura ha permitido que estudiantes de todas las regiones del país tengan acceso al aprendizaje del inglés como lengua extranjera y al mismo tiempo se capaciten en metodología de la enseñanza de la misma tanto en ambientes virtuales como en ambientes presenciales de aprendizaje. Es un programa $100 \%$ virtual y cuenta con material bibliográfico de reconocidas editoriales y con diseños propios para el aprendizaje online de la lengua.

\section{Número estudiantes 2011-II / 2013-II}

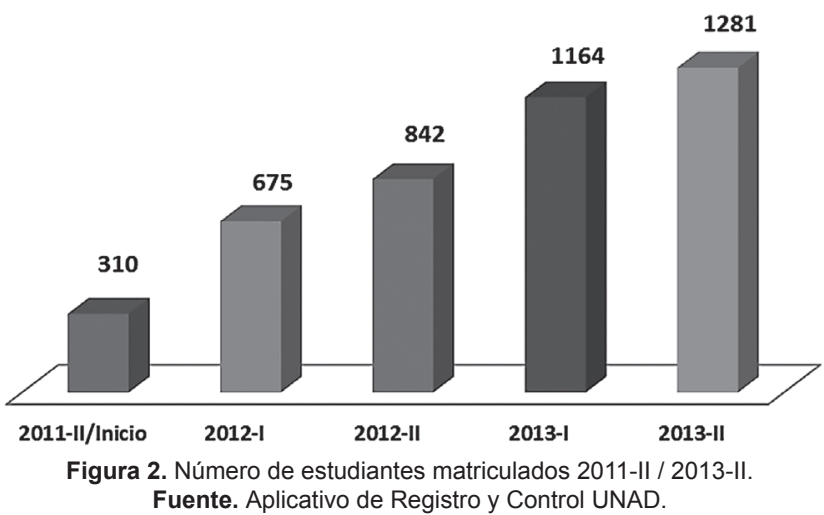

Propósito de formación. La Licenciatura en inglés como lengua extranjera está enfocada al desarrollo de competencias pedagógicas, investigativas, evaluativas, de gestión educativa y de liderazgo, a fin de lograr una educación crítica, reflexiva y comunitaria en función de mejorar la calidad de los procesos y resultados de aprendizaje; a la generación de innovación educativa desde la evaluación y las experiencias pedagógicas; a la investigación como eje articulador para la resolución de problemáticas contextualizadas en las diferentes regiones del país, con alto nivel de competencia pedagógica para su desempeño en procesos de aprendizaje mediado por TIC dentro del modelo de educación a distancia.

Misión. La Licenciatura en inglés como lengua extranjera tiene como misión formar docentes líderes con alta competencia comunicativa e intercultural, capaces de desempeñar su rol desde la investigación, la práctica pedagógica, las innovaciones metodológicas y didácticas con la utilización de las tecnologías de la información y de las comunicaciones, fortalecidos en el sentido humano y social que contribuirá a la formación integral de los ciudadanos colombianos en las diferentes comunidades locales, regionales y globales con calidad, eficiencia y equidad social.

Visión. Para el año 2019, la Licenciatura de Inglés como lengua extranjera será el programa líder, pionero e innovador en la formación de formadores de 
jóvenes y adultos en inglés como lengua extranjera a partir de la construcción de comunidades y redes académicas, acorde con las necesidades del país, los direccionamientos del MEN, los principios, fines y valores emanados en el PAP Solidario y la Escuela Ciencias de la Educación; reconocido a nivel nacional e internacional por la aplicación de las TIC para proceso enseñanza-aprendizaje del inglés como lengua extranjera, al igual que por sus aportes al mejoramiento de la educación en Latinoamérica, en particular Colombia y su plan de bilingüismo.

Créditos académicos. Este programa cuenta con una intensidad de 160 créditos, de los cuales el 75\% (120 créditos) son del ciclo básico obligatorio (común, disciplinar y profesional específico); el 25\% restante (40 créditos), corresponde a cursos electivos que el estudiante puede tomar de acuerdo con la oferta específica de la Licenciatura o a cursos afines al Programa dentro de la oferta académica de la UNAD.

\section{Proyección}

Una vez iniciada la oferta de la Licenciatura en Inglés como lengua extranjera, en junio de 2011, se determinó por parte de la Coordinación Nacional del Programa, la posibilidad de continuar con el proceso investigativo; fue así como desde el grupo Virtualex, en diciembre 2011, se presentó y avaló la propuesta SIGI-0092011: «Caracterización de los perfiles, competencias y niveles de inglés con los que ingresan los estudiantes a la Licenciatura en inglés como lengua extranjera de la Universidad Nacional Abierta y a Distancia».

Desde esta nueva investigación, se pretende, entre otros, definir las características demográficas, socio-económicas, laborales y académicas de los estudiantes de primer periodo académico del Programa, identificar las motivaciones y expectativas de ingreso a la LILE-UNAD, analizar las competencias y niveles de inglés con que ingresan los estudiantes a la Licenciatura e identificar las condiciones, instituciones y/o contextos en los que los estudiantes han aprendido la lengua inglesa. Lo anterior con el fin de generar y consolidar estrategias que permitan alcanzar altos estándares de calidad en la oferta de la Licenciatura en inglés como lengua extranjera de la UNAD, que redundará en la calidad académica e investigativa de los futuros docentes de inglés en Colombia.

En 2013-2, se inició el primer proceso de autoevaluación de la Licenciatura desde la participación en el Proyecto de Fortalecimiento a Licenciaturas en Idiomas del Ministerio de Educación Nacional y desde el marco de la Autoevaluación y Acreditación UNAD 2014. Como resultado de la participación en estos programas, se realizará un plan de acción o plan de mejoramiento que permitirá que la Licenciatura se actualice de manera permanente e innove en sus procesos académicos y curriculares. 


\section{Referencias bibliográficas}

Aebli, H. 2001. Factores de la enseñanza que favorecen el aprendizaje autónomo. Madrid: Narcea, S.A.

Anderson, T. 1996. The Movement and the Sixties. New York: Oxford University Press.

Castro, M. 2006. Guía de autoaprendizaje y metodología de estudio. Bogotá: Corporación Universitaria de Investigación y Desarrollo.

Council for Cultural Cooperation. Education Committee. Language and Policy Division. 2002. Marco Común Europeo de Referencia para las Lenguas. Aprendizaje y Evaluación. Traducción:Instituto Cervantes para la Traducción en Español, Madrid, 267 págs.

Díaz, F. \& Hernández, G. 1999. Estrategias docentes para un aprendizaje significativo, México: McGraw Hill.

Elkind, D. 2004. The Problem with Constructivism. The Educational Forum. Vol. 68 (No.4): 306-312. Disponible en: http://www.tandfonline.com/doi/ pdf/10.1080/00131720508984692\#.VHNrw7ctDIU Recuperado el 10 de febrero de 2013.

Fernández de Alaiza, B. 2000. La interdisciplinariedad como base de una estrategia para el perfeccionamiento del diseño curricular de una carrera de ciencias técnicas y su aplicación a la Ingeniería en Automática en la República de Cuba. Tesis como opción al Grado Científico de Doctor en Ciencias Pedagógicas. La Habana: Universidad Pedagógica Enrique José Varona.

Kant, I. 2002. Lecciones de ética. Barcelona: Crítica.

Knowles, M. 1970. The Modern Practice of Adult Education. From Pedagogy to Andragogy. Cambridge: Prentice Hall.

Leal, J. 2007. Plan de desarrollo UNAD 2007 - 2011. Bogotá: Universidad Nacional Abierta y a Distancia.

Ministerio de Educación Nacional Colombia (MEN). 2010. Observatorio Laboral. Bogotá: MEN.

Ministerio de Educación Nacional Colombia (MEN). 2010. Programa Nacional de Bilingüismo. Informe preliminar. Bogotá: MEN.

Ministerio de Educación Nacional Colombia (MEN). 2006. Estándares Básicos de Competencias en Lenguas Extranjeras: Inglés- Formar en lenguas extranjeras, el retoRevolución Educativa. Serie Guía 22. Bogotá: MEN. 
Ministerio de Educación Nacional Colombia (MEN). 2005. Bases para una nación bilingüe y competitiva. En: Al tablero No. 37. Octubre-diciembre 2005, 5 págs.

Ministerio de Educación Nacional Colombia (MEN). 1994. Ley 115 o Ley General de Educación.

Null, J. 2004. Is Constructivism Traditional? Historical and Practical Perspectives on a Popular Advocacy. En: The Educational Forum, Vol. 68 (No. 2): 180-188. Disponible en: http://www.researchgate.net/publication/249054120_Is_Constructivism_ Traditional_Historical_and_Practical_Perspectives_on_a_Popular_Advocacy. Recuperado el 10 de febrero de 2012.

Piaget, J. 1950. La epistemología de las relaciones interdisciplinarias. En: Interdisciplinariedad. Problemas de la enseñanza y de la investigación en las universidades, de Leo Apostel, G. Bergerr, A. Briggs y G. Michaud. Traducido por Francisco J. González Ortiz, 153-171, México: UNAM - Anuies, 1979.Universidad Nacional Abierta y a Distancia. 2011. Proyecto Académico Pedagógico Solidario Versión 3.0. Bogotá: UNAD.

Rodríguez, J. \& Sáenz, O. 1995. Tecnología educativa. Nuevas tecnologías aplicadas a la educación. Alcoy: Marfil.

Román, M. 1999. Currículum y evaluación: Diseños curriculares aplicados. Madrid: Complutense.

Román, M. 1998. El currículum y su evolución desde modelos de enseñanza-aprendizaje a modelos de aprendizaje-enseñanza. Una visión diacrónica y prospectiva. En: EducareEducere. Vol. 5. Págs. 9-57.

Román, M. \& Díez, E. 2000. El currículum como desarrollo de procesos cognitivos y afectivos. En: Revista Enfoques Educacionales, Vol.2 (2). Págs 48-67. ISSN 0717-3229

Román, M. y Díez, E. 1994. Currículum y enseñanza. Una didáctica centrada en procesos. Madrid: EOS.

Rousseau, J. 2005. Emilio o de la educación. Madrid. ISBN: 9788420635040 Filosofía: Alianza Editorial. 776 págs.Tobón, S. 2006. Formación basada en competencias. Pensamiento complejo, diseño curricular y didáctica. Bogotá: Banco de la República Biblioteca Luis Ángel Arango.

Universidad Nacional Abierta y a Distancia. 2010. Documento Maestro Licenciatura en Inglés como Lengua Extranjera. Bogotá: UNAD-Escuela Ciencias de la Educación. Universidad Nacional Abierta y a Distancia. 2008. Proyecto Académico Pedagógico Solidario. Bogotá: UNAD. 